\title{
Cardiovascular complications after radiotherapy
}

\author{
Izabela Nabiałek-Trojanowska ${ }^{1,2}$, Ewa Lewicka ${ }^{2}$, Anna Wrona ${ }^{3}$, Anna M. Kaleta ${ }^{2}$, \\ Zuzanna Lewicka-Potocka ${ }^{1,2}$, Grzegorz Raczak², Rafał Dziadziuszko ${ }^{3}$ \\ ${ }^{1}$ First Department of Cardiology, Medical University of Gdansk, Poland \\ ${ }^{2}$ Department of Cardiology and Electrotherapy, Medical University of Gdansk, Poland \\ ${ }^{3}$ Department of Oncology and Radiotherapy, Medical University of Gdansk, Poland
}

\begin{abstract}
Over the past decades, effective cancer therapies have resulted in a significant improvement in the survival rates for a number of cancers and an increase in the number of cancer survivors. Radiation therapy is widely used in the treatment of cancer, and it can induce various cardiotoxicities that differ considerably from chemotherapy-induced cardiotoxicity. They occur primarily as late radiation-induced complications, several years from the end of anticancer treatment and present as coronary artery disease, heart failure, pericardial disease, valvular heart disease and arrhythmias. Patients who recovered from cancer disease suffer from cardiac complications of anticancer treatment, it affects the quality of their lives and life expectancy, especially if the diagnosis is delayed. These patients may present distinct symptoms of cardiac injury, resulting from radiation-induced neurotoxicity and altered pain perception, which makes diagnosis difficult. This review highlights the need for a screening programme for patients who have undergone radiation therapy and which will subsequently have a potentially profound impact on morbidity and mortality. (Cardiol J 2020; 27, 6: 836-847)
\end{abstract}

Key words: radiotherapy, ionizing radiation, radiation injuries, cardiotoxicity, neoplasms

\section{Introduction}

Radiotherapy, along with surgery and chemotherapy, is a therapeutic technique used for definitive and palliative treatment of cancer. Currently, radiotherapy is a useful tool for the treatment of breast cancer, mediastinal lymphomas, head and neck tumors, and cancers of the lung, oesophagus, thyroid gland, prostate, and the genitals [1]. Cardiovascular complications resulting from radiotherapy were first noted in the 1970s. In 1978, a study in a group of 46 patients who underwent chest radiation revealed that radiotherapy led to cardiac fibrosis (involving the endocardium, myocardium, and pericardium) which mostly manifested clinically with pericarditis [2]. An association between ionizing radiation doses delivered to the heart and cardiac injuries were first noted in 1983. At the same time, a need to reduce the radiation doses applied was emphasized [3]. Studies indicated that the patient survival rate after effective anticancer treatment depended on late complications of the therapy delivered [4]. In patients with Hodgkin lymphoma who underwent radiotherapy, the most common causes of death included a primary or secondary cancer and cardiovascular disease (CVD) $[5,6]$.

At the present time, established radiationrelated cardiovascular complications include coronary artery disease (CAD), valvular heart disease, pericardial disease, heart failure (HF), right ventricular (RV) injury, arrhythmias, peripheral arterial disease, systemic hypertension, pulmonary hypertension, and thromboembolic disease [7]. These findings are the result of long-term follow-up of patients with a history of cancer with a relatively good prognosis for survival, particularly

Address for correspondence: Izabela Nabiałek-Trojanowska, MD, Department of Cardiology, Medical University of Gdansk, ul. Dębinki 7, 80-952 Gdańsk, Poland, tel: +48 781132796, +48 5834939 10, fax: +48 5834939 20, e-mail: izabela.nabialek@gumed.edu.pl

Received: 14.06.2018 Accepted: 11.10.2018 
Hodgkin lymphoma and early-stage breast cancer [8, 9]. A meta-analysis of 25 studies that included patients who received anthracyclines as anticancer treatment in childhood revealed that adding radiotherapy increased the rate of asymptomatic systolic $\mathrm{HF}$ [10]. Similarly, an analysis of adult patients who received anthracyclines for the treatment of leftside breast cancer showed that adding radiation therapy increased the risk of HF, which occurred in $0.5 \%$ of all patients and in $2.6 \%$ of those who received chemotherapy combined with radiation therapy [11].

The mechanism of radiation-related cardiac injury is early acute inflammation of small and medium-sized vessels, with cardiomyocytes necrosis due to hypoxia as a result of microvascular damage and interstitial fibrosis. Reactive oxygen species (ROS) produced in irradiated cells play an important role in cardiac injury, and they may damage cellular membrane proteins and lipids [12-16].

The rate of radiation-related cardiovascular complications depends on several additional risk factors, such as total radiation dose (significantly higher for doses above $30 \mathrm{~Gy}$ ), fractional radiation doses higher than 2 Gy per day, radiation doses delivered to the heart, the heart volume exposed to radiation, no shielding during radiotherapy, younger age at diagnosis, adjuvant chemotherapy (dependent on the total anthracycline dose), previous CVD, and cardiovascular risk factors [17, 18]. According to the American Society of Clinical Oncology, radiation-related cardiovascular complications develop in $10-30 \%$ of patients at 5 to 10 years after treatment [19].

For the last three decades, advances in radiation techniques have led to a reduction in the rates of radiotherapy complications. This was achieved by appropriate treatment planning with the use of three-dimensional (3D) imaging techniques, modern conformal radiation techniques with radiation beam intensity modulation (IMRT), and in the case of Hodgkin lymphoma, with a reduction of irradiated areas by radiation delivery only to involved fields (IFRT) or involved nodes (INRT). These methods allow for a reduction in the heart volume exposed to radiation $[19,20]$.

Radiation dose to the heart is now strictly controlled in radiotherapy planning systems, and details of radiation exposure are available for treating radiation oncologists in precise dose-volume histogram evaluations. Exact dose to each structure of the heart can also be visualized on each scan of planning computed tomography (CT), allowing for better prediction of early and late toxicity. Due to this progress and an understanding of the association between radiation dose and late cardiac complications of radiotherapy, doses to the heart are now much lower than in the past. In the three largest groups of patients treated with radiotherapy to the chest, typical mean doses to the heart for patients with breast cancer are in the range 1-3 Gy, for patients with lymphomas in the range of 1-10 Gy, and for patients with lung cancer, 1-20 Gy. These doses depend primarily on the anatomical location and stage of the tumor, as well as radiation techniques available in treatment facilities.

\section{Coronary artery disease}

Exposing the heart to ionizing radiation during anticancer therapy increases the risk of CAD. Damage to nerve endings caused by neurotoxicity of radiation therapy in the radiated fields is a factor impeding early diagnosis of CAD due to reduction of chest pain sensation in this group of patients. The risk of CAD increases with the radiation dose administered to the heart [21, 22]. The mechanism of radiation-related injury to coronary arteries is multifactorial and includes endothelial damage, atherosclerotic plaque rupture, thrombosis, and vasospasm $[7,12]$. Coronary artery lesions associated with radiotherapy are typically located in the ostia and proximal vessel segments. For left- and right-sided breast cancer irradiation they mostly develop in the left anterior descending artery and right coronary artery, respectively, and in the left main coronary artery, the left circumflex artery, and the right coronary artery after mediastinal radiotherapy for Hodgkin lymphoma, corresponding to the areas exposed to radiation $[7,17,23$, 24]. In addition, myocardial perfusion defects are seen regardless of the coronary artery territories, which suggests microvascular damage. This was shown using technetium-99m sestamibi myocardial perfusion scintigraphy in $50 \%$ of the observed patients at 1 year following adjuvant radiotherapy for left-sided breast cancer [25].

Modern radiotherapy techniques are still being developed, which creates an opportunity to modify and minimize radiation doses delivered to normal tissue, thus reducing the rates of cardiovascular complications. At the present time, radiotherapy is based on 3D or 4D (accounting for respiratory motion) treatment planning with dose-volume histograms, depicting dose distribution in predefined anatomical areas of the heart. It is possible to delineate the contours of the pericardium and coronary arteries with the available imaging tech- 
niques, such as CT or magnetic resonance imaging (MRI). As a result, it is possible to estimate the radiation dose delivered to the whole heart, coronary arteries, atria, ventricles and cardiac conduction system [26, 27].

Progression of CAD in these patients may vary. The disease may occur early, with symptoms of acute coronary syndrome and even sudden cardiac death, but it usually develops slowly and is detected approximately 15 years after treatment. A study based on the observation of 34,825 patients with breast cancer treated with radiotherapy in Sweden and Denmark in 1976-2006 showed that angina and myocardial infarction (MI) occurred with a higher rate in patients irradiated to the left breast compared to those irradiated to the right breast. Furthermore, the radiation dose to the whole heart was on average 6.3 Gy during left-sided radiotherapy and 2.7 Gy during right-sided radiotherapy, showing a relation between the radiation dose delivered to the heart and incident CAD [21]. CAD was found in $10.4 \%$ of patients followed for at least 20 years after mediastinal radiotherapy with the radiation dose ranging from 25 to 42 Gy [28].

A study performed on an animal model indicated that radiation therapy accelerated the development of coronary artery atherosclerosis related to hypercholesterolemia [29]. In addition, observation of a large population of patients after treatment for Hodgkin lymphoma revealed that angina, $\mathrm{MI}$, and $\mathrm{HF}$ occurred at a higher rate in patients with hypercholesterolemia, diabetes mellitus, and a history of smoking. This analysis also showed that angina and $\mathrm{HF}$ occurred more frequently in patients treated at a younger age, below 20 years $[6,30]$. A retrospective analysis of patients who underwent radiotherapy for Hodgkin lymphoma revealed that a higher risk of late cardiovascular complications of irradiation showed an association with a younger age, male sex, radiation dose delivered to the whole heart, and dose inhomogeneity [31]. Higher radiation dose homogeneity may be obtained using modern radiotherapy techniques and is associated with a lower risk of cardiac damage for the same radiation dose delivered to the heart. It is an important parameter when planning treatment with ionizing radiation.

In primary prevention of progressive coronary disease each patient after chest radiotherapy should be screened for classic cardiovascular risk factors, with adequate management and correction of modifiable risk factors. A model of screening for CAD depends on the Systemic Coronary Risk Estimation (SCORE) result and accompanying symptoms, and among cancer survivors, it does not differ from the general population [32, 33]. The main issue is the time to start a screening program, because radiotherapy leads to premature onset of CAD mainly in asymptomatic patients. A study on Hodgkin disease survivors, with no symptoms of CAD revealed left ventricular (LV) segment hypokinesis in rest echocardiography in $17 \%$ of patients who have had mediastinal radiation therapy with high doses of radiation (more than 35 Gy) [34].

A recent expert consensus statement from European Association of Cardiovascular Imaging and the American Society of Echocardiography [17] recommends echocardiographic evaluation in asymptomatic high-risk patients starting 5 years after radiation exposure and 10 years after exposure in the others, with reassessment every 5 years. According to this document, high-risk patients should also receive a functional non-invasive stress test for CAD detection within 5 to 10 years after completion of chest irradiation. This strategy was adopted in the present center, and as treadmill exercise electrocardiograms (ECGs) may not reflect the burden of CAD, patients who are at least 5 years after radiotherapy are referred for stress echocardiography (including the assessment of changes in LV global longitudinal strain).

Apart from stress echocardiography, either exercise or dobutamine, perfusion single-photon emission CT and MRI may reveal stress-induced LV wall motion abnormality [17]. For younger patients coronary CT angiography is a valuable option because of its high negative predictive value. Exposure to radiation is still an issue, however modern CT scanners allow for dose reduction. Another limitation of coronary CT angiography are advanced calcifications, which if significant may impede detection of coronary arteries stenosis [35]. Cardiac MRI is an excellent method for reliable assessment of cardiac structure and function, but it poses significant logistic and economic challenges.

Pharmacological treatment of CAD does not differ from the general population, it includes acetylsalicylic acid or double antiplatelet therapy after stent implantation, statin, beta-blocker and angiotensin-converting enzyme (ACE) inhibitor or angiotensin receptor blocker (ARB), if not contraindicated. Invasive treatment: percutaneous coronary intervention (PCI) with stent implantation is recommended in patients with acute coronary syndrome or stable CAD refractory to pharmacological therapy. Conservative treatment in these conditions leads to poorer prognosis [33]. 
In reference to cardiac surgery and coronary artery bypass grafting (CABG), some studies showed increased perioperative risk resulting from mediastinal fibrosis [36, 37], but new research indicates that previous radiotherapy does not increase surgical complications and does not impact long-term survival comparing to cancer-free patients in isolated CABG surgery. The utilization of internal thoracic artery for graft was reduced in the study group, which worsens outcome of CABG in the general population [38]. In patients who have undergone prior chest radiotherapy, CT should be performed before deciding on CABG to evaluate the degree of mediastinal fibrosis and potential calcifications in the ascending aorta and aortic arch, which could impede implantation of aortocoronary grafts. Moreover, regarding potential radiation damage to the internal thoracic artery, angiographic assessment of this artery should be performed. As valvular heart disease could also be a complication of radiotherapy, a detailed valve examination is necessary before CABG in order to avoid repeated sternotomy [38]. In a recent study, patients with prior mediastinal radiotherapy referred for surgical aortic valve replacement have had significantly worse long-term survival compared to a matched control group [37].

\section{Pericardial disease}

Currently, pericardial diseases occur less often as a complication of chest radiotherapy. These conditions include acute pericarditis, chronic pericarditis, chronic pericardial effusion, and constrictive pericarditis. They mostly develop in patients treated with a radiation dose of at least 50 Gy [39]. Treatment decisions depend on the type of pericardial disease and the clinical condition of the patient $[3,40]$.

Common symptoms of acute pericarditis include fever, chest pain related to body position and respiratory movements, pericardial effusion on echocardiography, and ST segment elevation and PR depression in multiple ECG leads. In a patient presenting with acute chest pain or elevated cardiac troponin level, it is necessary to rule out acute coronary syndrome by ECG and echocardiography with LV contractility assessment. Acute exudative pericarditis rarely occurs during radiotherapy, and it is related to inflammation and necrosis of a tumor located near the heart [17]. A study performed on an animal model showed that acute pericarditis developed within $6-48 \mathrm{~h}$ after irradiation with a 20-40 Gy dose [40]. Delayed acute pericarditis, either symptomatic or asymptomatic, may develop in $2-5 \%$ of patients at $2-145$ months after chest radiotherapy. This condition very rarely leads to cardiac tamponade which requires pericardial drainage [7].

Hemodynamically stable patients are usually managed medically, initially with non-steroidal anti-inflammatory drugs and colchicine, followed by glucocorticoids and immunosuppressive drugs if the first-line treatment is ineffective. Spontaneous clearance of pericardial effusion usually takes up to 2 years [17]. Data from patients treated with older radiotherapy techniques indicate that pericardial disease developed in $10-12 \%$ of patients at 6 to 18 months after irradiation, with acute pericarditis in $35 \%$ of these cases [3, 40].

Patients with impending or actual cardiac tamponade require invasive treatment. Percutaneous pericardiocentesis with extended catheter drainage is considered the safest method to remove excess pericardial fluid in cancer patients, even in those with thrombocytopenia [41, 42]. Surgical drainage is an alternative approach but is associated with a higher perioperative risk.

Chronic pericarditis may occur from 6 months to 15 years after completion of radiotherapy and develops in up to $20 \%$ of patients treated with high radiation doses. This process is associated with organization of fibrinous exudates, fibrous adhesions, and collagenous thickening, predominantly of the parietal pericardium. The incidence of pericardial layer thickening following radiation therapy increases with time, affecting $33 \%$ of patients after more than 20 years [28, 43]. Its occurrence depends on the radiation dose administered to the whole heart, including both the right and the left atrium $[24,44]$.

Constrictive pericarditis develops in $4 \%$ to $20 \%$ of radiotherapy patients, particularly those treated with older techniques, and it usually requires pericardiectomy $[17,43,45,46]$. In suspicion of constrictive pericarditis, constrictive cardiomyopathy should be ruled out by echocardiography, chest X-ray, CT or MRI revealing thickening of pericardium, calcifications, abnormal interventricular septum movement, abnormal mitral valve flow pattern, or by right heart catheterization if these tests are inconclusive [47].

In the case of pericardial effusion, and especially its recurrence, it may be problematic to determine whether it is caused by radiotherapy, tumor progression or infection. Biochemical and microbial blood sample tests, polymerase chain reaction $(\mathrm{PCR})$ are useful, but in some cases 
pericardial fluid examination may be required in recognizing pericarditis etiology. According to the newest European Society of Cardiology (ESC) guidelines regarding pericardial disease, diagnosing of etiology should be performed in patients, who present with fever $>38^{\circ} \mathrm{C}$, subacute development of symptoms, $>20 \mathrm{~mm}$ of fluid in imaging and low response to acetylsalicylic acid/non-steroid antiinflammatory drug treatment [48].

Imaging and comparing of effusion density in pericardial cavity may be helpful in recognizing etiology: protein-rich fluid, consisting of blood e.g. in aortic aneurysm rupture or aortic dissection; high density fluid in lymph leakage; pericardium thickening in inflammation or thickening with calcification in constrictive pericarditis. Imaging by CT or MRI may reveal direct neoplastic infiltration or metastasis. Positron emission tomography individually or combined with CT (recommended), is helpful in the diagnoses of neoplasmatic etiology of pericarditis if fluodexyglucose uptake in tumor cells is observed.

The most common primary cancer in pericardium is mesothelioma, and benign tumors are lipoma and fibroma. Secondary malignancy comes mostly from lung cancer, breast cancer, melanoma, lymphoma and leukemia. The presence of cancer cells in pericardial fluid is associated with worse outcomes in lung cancer patients but such an association was not found in breast cancer patients [42]. Generally, the utility of the assessment of cancer markers in serum and in pericardial fluid has still not been proven, but a positive result of e.g. EGFR mutation test in pericardial effusion in lung cancer influences the decision about targeted anticancer therapy implementation [47]. Pericardium biopsy allows for histological diagnosis of cancer. Among patients with recognized cancer, the spreading of malignancy is a reason of pericarditis in approximately $30 \%$ of patients [47].

In cancer etiology of pericarditis, intrapericardial administration of cytostatic or sclerotic agent may decrease a frequency of fluid recurrence. As a palliative treatment, radiotherapy or pleuropericardiotomia alleviates symptoms of recurrent pericarditis.

\section{Valvular heart disease}

Valvular heart disease complicating radiotherapy develops in $10 \%$ of patients. Radiation-induced valve abnormalities are characteristically distributed, with thickening and calcifications located at the basal and medial parts of the leaflets, while leaflet tips and commissures are spared, allowing distinction from rheumatic disease. Calcification of the mitro-aortic curtain, the junction between the anterior mitral leaflet valve and the aortic root, is also characteristic for post-radiation damage [49]. A retrospective analysis of post-radiotherapy patients with Hodgkin lymphoma showed valvular lesions in $6.2 \%$ patients after an average 22 years of observation, with aortic stenosis in more than a half of these patients [22]. However, in another study of patients followed up for at least 20 years after mediastinal radiotherapy with a minimal radiation dose of $35 \mathrm{~Gy}$, aortic stenosis was found in $16 \%$, mitral regurgitation in $60 \%$, and tricuspid regurgitation in $4 \%$ of patients in the study group [28]. Valvular lesions are more common on the left side of the heart than on the right side, independently of radiation dose [50]. In cases of severe valvular lesions requiring invasive treatment, transcatheter methods (e.g., transcatheter aortic valve implantation) are considered to be safer compared to conventional valve surgery due to mediastinal fibrosis and aortic calcifications which are common after radiotherapy.

\section{Left ventricular dysfunction}

Radiotherapy-induced cardiac damage leads to myocardial fibrosis $[39,51]$ which mostly results in LV diastolic dysfunction. The main mechanism underlying cardiomyocytes injury is microvascular damage. In addition, combining radiotherapy with cardiotoxic chemotherapy using anthracyclines, especially in young patients and in females with breast cancer, may also lead to LV systolic dysfunction. Advanced myocardial fibrosis may predispose to restrictive cardiomyopathy phenotype observed after radiation therapy, with the presence of severe diastolic dysfunction and symptoms of HF. A differential diagnosis from constrictive pericarditis is recommended by echocardiography, CT, MRI, and right heart catheterization, if required. The principle in diagnosis is reduced myocardial elasticity, that is due to impaired myocardial relaxation in restrictive cardiomyopathy and constricted chambers by pericardium with normal diastolic function in constrictive pericarditis. In restrictive cardiomyopathy transthoracic echocardiography reveals normal or thickened left and right ventricle, normal or reduced LV cavity, enlarged atria, restrictive transmitral filling pattern (E/A ratio $>2$ ), and reduced peak early-diastolic mitral-annular velocities in tissue Doppler $\left(\mathrm{e}^{\prime}<8 \mathrm{~cm} / \mathrm{s}\right)$ independent of respiration. Other imaging modalities, as CT or 
MRI may detect structural changes in myocardium e.g. fibrosis and unthickened pericardial layer. Cardiac catheterization reveals increased RV systolic pressure (above $50 \mathrm{mmHg}$ ), and $\mathrm{LV}$ end-diastolic pressure (LVEDP) by $5 \mathrm{mmHg}$ higher than RV end-diastolic pressure (RVEDP) [47].

Left ventricular systolic function is routinely assessed by LV ejection fraction (LVEF) measurement during echocardiography. The recommended method to evaluate LVEF is 3D echocardiography. If this method is not available, LVEF should be assessed by the 2D biplane Simpson method, based on contouring of the LV cavity during systole and diastole in the apical 4- and 2-chamber views. Disadvantages of this method include its dependence on the appropriate angle of transducer and the fact that it reveals relatively late impairment of LV systolic function.

A new method of an increasing significance is the assessment of LV global longitudinal strain (GLS) by $2 \mathrm{D}$ speckle tracking echocardiography (STE). In this technique, a percentage of cardiac muscle shortening in the longitudinal layer is assessed during one or three cardiac cycles. Newer software allows for distinguishing the inner and outer myocardial layers, what is important for diagnosis. Abnormalities within the inner (subendocardial) layer indicate mainly CAD etiology, but can be also present in patients with chemotherapy-induced cardiotoxicity. In turn, abnormalities within the outer (subepicardial) layer indicate myocardial inflammation, rarely cardiotoxicity. Apart from the longitudinal muscle layer, motion in the radial and circumferential myocardial layers may also be tracked by STE. These methods are also used for the assessment of RV function.

An analysis of patients after radiotherapy for chemotherapy-naive early stage of breast cancer showed a decrease in GLS and apical longitudinal strain after radiotherapy for left-sided breast cancer. In this group of patients, a compensatory increase in basal longitudinal strain was also observed. Other conventional echocardiographic parameters were not sensitive enough to show any changes of the LV systolic function. In patients after radiotherapy for right-sided breast cancer, speckle tracking analysis revealed decreased longitudinal strain in basal anterior segments [52]. The observed changes in longitudinal strain corresponded to the irradiated region of the heart. The study showed that assessment of longitudinal strain by STE is a more sensitive method in revealing LV systolic dysfunction compared to LVEF measurements and visual assessment of segmen- tal wall motion abnormalities. A meta-analysis of 16 studies that included patients with HF, acute MI, and valvular heart disease showed a superior predictive value of GLS assessment compared to LVEF for predicting major adverse cardiac events [53].

A study of patients with Hodgkin lymphoma treated with a radiation dose of at least $35 \mathrm{~Gy}$ in comparison to the Framingham study population showed a decrease in the LV fractional shortening to less than $30 \%$ in $36 \%$ of patients in the study group and in $3 \%$ of subjects in the Framingham study population [28]. An MRI study showed that late gadolinium enhancement corresponded to the radiation fields within the heart, and that delivery of a radiation dose to the heart had an effect on the occurrence of radiation-induced cardiomyopathy [54]. A study in breast cancer patients revealed that the increase in the serum high-sensitive troponin $\mathrm{T}$ level depended on the total radiation dose delivered to the whole heart and the LV. Long-term implications of this finding are unknown and require further studies [55].

In 2011, Polish National Team of Cardiologic and Oncologic Supervision published recommendations regarding care for breast cancer patients [56]. The emphasis was put on prevention, early diagnosis, by highly specific and sensitive methods, and treatment of CVD. The limit of significant LV dysfunction was set on a drop of LVEF of $15 \%$, to value less than $50 \%$.

According to the 2016 ESC guidelines [7], an LVEF decrease by means of echocardiography of more than $10 \%$ to a value below the lower limit of normal values (LVEF < 50\%) suggests cardiotoxicity. And a relative GLS reduction by more than $15 \%$ compared to the baseline may suggest the risk of cardiotoxicity. Both guidelines recommended ACE inhibitor therapy in patients with LVEF lower than $50 \%$. Newer guidelines added monitoring of B-type natriuretic (BNP) peptide or NT-pro-BNP concentration to evaluate cardiac dysfunction and make decisions on starting pharmacological cardiovascular therapy or changing anticancer treatment.

These guidelines refer to complications of chemotherapeutic agents, and it should be noted that specific guidelines regarding radiation-induced heart disease are lacking. According to the 2017 American Society of Clinical Oncology (ASCO) guidelines, patients who were exposed to at least 30 Gy of irradiation containing the heart in the irradiated field, or those who underwent radiotherapy with less than $30 \mathrm{~Gy}$ with the heart in the irradiated 
field but combined with anthracycline treatment are at increased risk of cardiac dysfunction [57].

If LV systolic dysfunction is detected, it is managed medically similar to other etiologies of HF, mostly with beta-blocker, ACE inhibitors or ARBs [51]. In patients with end-stage HF who underwent radiotherapy, the previous radiation therapy is not a contraindication to cardiac transplantation. Despite technical difficulties in performing the surgery due to severe mediastinal fibrosis in the irradiated regions, the survival rate after cardiac transplantation does not differ significantly from life expectancy after cardiac transplantation in radiotherapy-naive patients [36].

\section{Right ventricular dysfunction}

The RV is located immediately behind the anterior chest wall and thus it is most prone to the adverse effects of mediastinal radiotherapy. Although the latest cardiooncological guidelines mostly discuss LV function, it has been recently suggested that cardiovascular morbidity and mortality associated with oncological treatment is also related to the condition of the RV [58].

Radiation-induced RV damage is mainly caused by myocardial fibrosis and remodeling, damage to blood vessels, and accelerated coronary artery atherosclerosis [58]. In addition, radiotherapy-induced pericardial fibrosis may induce RV diastolic dysfunction followed by systolic dysfunction, as the RV is subjected to low afterload under normal conditions [59].

The risk factors for radiotherapy-induced damage to the RV include total and fractional radiation dose, irradiation method, tumor location, and concomitant diseases [58]. In breast cancer patients, the number of individuals with RV systolic dysfunction increases with time following treatment with anthracyclines, trastuzumab and/or radiotherapy [60]. Examination using the novel method of ultrasonic tissue characterization showed an increase in myocardial echogenicity related to the radiation dose. The study was performed in patients with left breast cancer and showed that the RV free wall was exposed to the highest radiation dose during radiotherapy [61]. The increase in $\mathrm{RV}$ free wall echogenicity was accompanied by a decreased tricuspid annular plane systolic excursion (TAPSE).

Right ventricular structure and function may be assessed by echocardiography and MRI. Echocardiographic parameters include TAPSE, RV fractional area change, lateral tricuspid annular systolic velocity (RV S'), RV free wall longitudinal strain, and RV wall thickness. The gold standard imaging tool is MRI which allows for precise evaluation of the RV structure and function including end-diastolic volume, end-systolic volume, and RV ejection fraction [62].

\section{Arrhythmias}

Electrocardiographic abnormalities and arrhythmias are recognized in $16-36 \%$ patients with a history of radiotherapy [7], including bradyarrhythmias, tachyarrhythmias, and various conduction disturbances. These arrhythmias are usually related to fibrosis involving the atria and the conduction system. An association was found between arrhythmic events and the radiation dose in the right atrium, the left atrium and the whole heart in patients treated with high dose radiation therapy for non-small-cell lung cancer [44].

Chemotherapy combined with radiotherapy may increase the arrhythmic risk. Many chemotherapeutic agents lead to the QTc interval prolongation (in particular arsenic trioxide, tyrosine kinase inhibitors, and doxorubicin). Electrolyte disturbances which are common during anticancer treatment (both primary and secondary to vomiting, diarrhoea and skin lesions after radiotherapy) and administration of other drugs that increase the QTc interval (antiemetics, antibiotics, antimycotics, psychotropic agents, and antiarrhythmic drugs) also favour arrhythmic events [7, 28].

Atrial fibrillation (AF) is the most common supraventricular arrhythmia induced by radiotherapy, chemotherapy and/or surgical treatment. Indications for chronic oral anticoagulation have to be considered in patients with AF, which may be problematic due to an elevated risk of both thromboembolic events and bleeding complications in patients with malignancies. Low molecular weight heparins, vitamin $\mathrm{K}$ antagonists, and novel oral anticoagulants may be used for anticoagulation $[63,64]$. It should be noted that the $\mathrm{CHA}_{2} \mathrm{DS}_{2}$-VASc and HAS-BLED risk scores to evaluate the risk of thromboembolism and bleeding complications, respectively, have not been validated in cancer patients.

Radiotherapy-induced ventricular arrhythmias are caused by myocardial ischemia and LV dysfunction [7]. Similarly, sinus node dysfunction and atrioventricular conduction disturbances are the result of ischemia, myocardial fibrosis, and direct damage to the cardiac conduction system caused by irradiation $[12,43]$. Right bundle branch block is most frequently seen after chest radiotherapy 
because the right bundle is situated just under the endocardium and is most susceptible to irradiationinduced damage [16].

In summary, factors favouring the occurrence of arrhythmia such as previous heart disease, QTc prolonging drugs, electrolyte disturbances, kidney function, thyroid hormone level, and impaired hepatic metabolism have to be taken into consideration in patients undergoing radiotherapy. Serious arrhythmias and conduction disturbances need to be treated by either drug therapy or cardiac device implantation.

\section{Peripheral arterial disease}

In addition to the effect on the heart, radiotherapy delivered to the neck and mediastinum may also damage blood vessels located in the irradiated area. Mechanisms include direct damage to vessel walls, injury of vasa vasorum, fibrosis, and accelerated atherosclerosis [65]. Significant atherosclerotic lesions in either carotid or subclavian arteries were observed in $7.4 \%$ of patients at 17 years after radiation therapy. The average patient age at the time these vascular lesions were diagnosed was 34 years, and stroke or a transient ischemic attack occurred at an average age of 51 years [22]. Pathological lesions in femoral arteries developed not earlier than 5 years after radiotherapy of the abdominal and pelvic regions [66, 67]. In addition, irradiation of the abdominal cavity may lead to the development of arterial hypertension mediated by damage to renal vessels.

The severity of arterial damage depends on radiation dose and time since treatment [68]. It was also shown that conventional CAD risk factors including hypertension and hypercholesterolemia have a synergistic effect on the development of radiation-induced arterial disease, and this risk may be minimized with proper treatment [67]. Radiation-induced arterial disease is managed using the same approach as atherosclerotic disease, and the treatment includes use of acetylsalicylic acid, statin, and percutaneous and surgical interventions. Invasive treatment outcomes are similar in patients with or without a history of malignancy [66].

\section{Arterial hypertension and orthostatic hypotension}

Irradiation of the head or neck may result in disruption of baroreflex, which in the chronic phase is characterized by severe labile blood pressure (BP), and this condition is commonly referred to as the syndrome of baroreflex failure. The baroreflex normally functions via carotid stretch receptors to maintain heart rate and BP. The mechanism of radiation-induced baroreflex failure is direct damage of carotid sinus receptors and their afferent vagal branches or accelerated atherosclerosis and fibrosis within arterial walls. Decreased baroreflex sensitivity and inefficient inhibitory activity may result in increased sympathetic tone. More rarely, inadequate efferent baroreceptor activity may lead to orthostatic hypotension with syncope, BP drop during sleep and bradycardia.

Baroreflex failure syndrome presents with headache, anxiety, emotional lability, tachycardia, hypertension, orthostatic lightheadedness, and/or hypotensive episodes. There may be severe BP elevation, even exceeding $250 \mathrm{mmHg}$, which may cause complications such as cerebral hemorrhage or encephalopathy. The onset of increased BP may occur days or weeks from completion of radiotherapy [69]. In differential diagnosis tests to rule out secondary causes of hypertension (e.g., renal artery stenosis or parenchymal disease, pheochromocytoma, other endocrinopathies) should be performed.

Cardiovascular dysfunction in these patients can be detected by ambulatory BP monitoring demonstrating abnormalities in resting BP and increased BP variability. To confirm baroreflex failure, deep breathing, Valsalva maneuver and tests with intravenous administration of phenylephrine or sodium nitroprusside show abnormal BP pattern and a lack of reflex brady- and tachycardia in response to $\mathrm{BP}$ changes.

In acute phase primary treatment is antihypertensive therapy and in chronic phase inhibition of central noradrenergic neurotransmission (e.g. clonidine) is recommended. Orthostatic and postexercise hypotension resulting from baroreflex disorder may be challenging in management of hypertension. Implantation of pacemaker should be considered in patients with malignant bradycardia due to vagotonia, and supplementation of fludrocortisone and dietary salt in case of hypotension [69-75].

Impaired cardio-autonomic functions which were not apparent clinically were shown by Goyal et al. [72] in neck irradiated patients due to cancer disease. The authors evaluated heart rate variability with time domain analysis of 5 min ECG recording. Postural cardiovascular reflexes were studied with changes in $\mathrm{BP}$ and heart rate with the lying to standing test. The present study revealed a reduction in overall time domain measures of heart rate variability and weakened postural reflexes in 
neck irradiated patients. Whether decreased heart rate variability in neck irradiated patients reflects an independent risk of cardiovascular morbidity requires further investigation. Nevertheless, the dose of radiation delivered to the carotid sinus should be monitored and restricted, as well as radiation oncologists should be aware of baroreflex failure syndrome, as they often could be the first to diagnose it and detect it early.

\section{Summary}

Ongoing advances in oncology and treatment of malignant hematological diseases lead to the development of new therapeutic options that increase the proportion of patients with long-term survival chances. This increase in life expectancy of cancer survivors has led to an increased incidence of long-term complications of anticancer therapy, including adverse cardiovascular effects of radiotherapy. The aims of cardiooncology include the prevention, diagnosis, and treatment of CVDs in the population of oncological patients $[7,62,76]$. For patients treated with radiation therapy, there is a need for accurate recommendations regarding the planning and extent of cardiac screening for early diagnosis and effective treatment of cardiovascular complications of radiotherapy $[5,77,78]$.

Currently, available data are provided from breast cancer and Hodgkin lymphoma groups, who reach relatively long-term survival. It is necessary to identify groups of patients with higher risk of radiation-induced cardiovascular complications and prepare for them a follow-up plan. It should be taken into consideration that assessment of cardiovascular risk based on the radiation dose delivered to the heart or the volume of the irradiated heart by comparing groups of patients treated with older and newer methods of radiotherapy is difficult, because of relevant differences in radiation techniques. Anyway, according to ASCO guidelines based on meta-analysis of available research, minimizing doses delivered to heart and volume of the irradiated heart is an essential step in the prevention of long-term complications of radiotherapy [57].

In patients who have undergone chest radiation therapy, evaluation based on signs and symptoms and echocardiographic surveillance should be implemented, starting 5 years after treatment in high-risk patients and 10 years in all other patients. Further reassessment should be performed every 5 years. Even if asymptomatic, high-risk patients should also be referred for functional non-invasive stress tests within 5 to 10 years after completing irradiation therapy [17, 42]. Pregnant women and those who are planning pregnancy should be carefully monitored, as gestation may unmask subclinical cardiotoxicity [35].

Another important issue is the treatment of comorbidities and reduction of modifiable cardiovascular risk factors necessary among those patients. According to the ESC guidelines regarding cardiovascular prevention for cancer survivors, recommendations include healthy diet, smoking cessation, reduction of body weight and regular aerobic exercises, which are especially helpful in prevention and treatment of cardiotoxicity [79].

\section{Conflict of interest: None declared}

\section{References}

1. Gustavsson A, Osterman B, Cavallin-Ståhl E. A systematic overview of radiation therapy effects in non-Hodgkin's lymphoma. Acta Oncol (Madr). 2003; 42(5-6): 605-619, indexed in Pubmed: 14596518.

2. Di Mattéo J, Vacheron A, Heulin A, et al. [Cardiac complications of thoracic radiotherapy]. Arch Mal Coeur Vaiss. 1978; 71(4): 447-452, indexed in Pubmed: 96762.

3. Vacheron A, Heulin A, Baubion N, et al. Cardiac complications of radiotherapy. Ann Cardiol Angeiol (Paris) [Internet. 1983; 32(7): 465-472, indexed in Pubmed: 6660823.

4. Cuzick J, Stewart HJ, Peto R, et al. Overview of randomized trials of postoperative adjuvant radiotherapy in breast cancer. Cancer Clinical Trials. 1987; 71(1): 7-14, indexed in Pubmed: 3539330.

5. Toltz A, Shin N, Mitrou E, et al. Late radiation toxicity in Hodgkin lymphoma patients: proton therapy's potential. J Appl Clin Med Phys. 2015; 16(5): 167-178, doi: 10.1120/jacmp.v16i5.5386, indexed in Pubmed: 26699298.

6. Aleman BMP, van den Belt-Dusebout AW, De Bruin ML, et al. Late cardiotoxicity after treatment for Hodgkin lymphoma. Blood. 2007; 109(5): 1878-1886, doi: 10.1182/blood-2006-07-034405, indexed in Pubmed: 17119114.

7. Zamorano JL, Lancellotti P, Rodriguez Muñoz D, et al. 2016 ESC Position Paper on cancer treatments and cardiovascular toxicity developed under the auspices of the ESC Committee for Practice Guidelines: The Task Force for cancer treatments and cardiovascular toxicity of the European Society of Cardiology (ESC). Eur Heart J. 2016; 37(36): 2768-2801, doi: 10.1093/eurheartj/ ehw211, indexed in Pubmed: 27567406.

8. Gaya AM, Ashford R. Cardiac complications of radiation therapy. Clin Oncol. 2005; 17(3): 153-159, doi: 10.1016/j.clon.2004.09.016.

9. Darby SC, Ewertz M, McGale P, et al. Risk of ischemic heart disease in women after radiotherapy for breast cancer. N Engl J Med. 2013; 368(11): 987-998, doi: 10.1056/NEJMoa1209825, indexed in Pubmed: 23484825.

10. Kremer L, Pal Hv, Offringa M, et al. Frequency and risk factors of subclinical cardiotoxicity after anthracycline therapy in children: a systematic review. Ann Oncol. 2002; 13(6): 819-829, doi: 10.1093/annonc/mdf167.

11. Valagussa P, Zambetti M, Biasi S, et al. Cardiac effects following adjuvant chemotherapy and breast irradiation in operable breast 
cancer. Ann Oncol. 1994; 5(3): 209-216, doi: 10.1093/oxfordjournals.annonc.a058795, indexed in Pubmed: 8186169.

12. Wrona A, Dziadziuszko R, Jassem J. Red.) Szymański F, Filipiak K, Warszawa IP 2017: 137-158. Radioterapia a ryzyko powikłań ze strony ukladu sercowo-naczyniowego. Nieklasyczne czynniki ryzyka chorób układu sercowo-naczyniowego w gabinecie lekarza praktyka [Internet]. ITEM Publishing 2017: 137-158. Available from: http://bibliografia.gumed.edu.pl/cgi-bin/expertus. exe?KAT $=$ f $\% 3 \mathrm{~A} \% 5$ Chidden $\% 5$ Cexpertus $\% 5$ parametr. $02 \% 5 \mathrm{C}$ $\& \mathrm{FST}=$ data.fst $\& \mathrm{FDT}=$ data.fdt $\&$ ekran $=\mathrm{ISO} \& \operatorname{lnkmsk}=2 \& \mathrm{con}$ $\mathrm{d}=$ AND\&mask $=2 \& \mathrm{~F} \_00=06 \& \mathrm{~V} \_00=$ Nieklasyczne + czynniki + ryzyka + chor\%F3b + uk\%B3adu + sercowo-naczyniowego $+\mathrm{w}$ + gabinecie +1 .

13. Stewart J, Fajardo L, Gillette S, et al. Radiation injury to the heart. Int J Radiation Oncol *Biology* Physics. 1995; 31(5): 1205-1211, doi: 10.1016/0360-3016(94)00656-6.

14. Rodemann H, Bamberg M. Cellular basis of radiation-induced fibrosis. Radiotherapy Oncol. 1995; 35(2): 83-90, doi: 10.1016/0167-8140(95)01540-w, indexed in Pubmed: 7569029 .

15. Hendry JH, Akahoshi M, Wang LiS, et al. Radiation-induced cardiovascular injury. Radiat Environ Biophys. 2008; 47(2): 189-193, doi: 10.1007/s00411-007-0155-7, indexed in Pubmed: 18193445.

16. Bhattacharya S, Asaithamby A. Ionizing radiation and heart risks. Semin Cell Dev Biol. 2016; 58: 14-25, doi: 10.1016/j. semcdb.2016.01.045, indexed in Pubmed: 26849909.

17. Lancellotti P, Nkomo VT, Bergler-Klein J, et al. Expert consensus for multi-modality imaging evaluation of cardiovascular complications of radiotherapy in adults: a report from the European Association of Cardiovascular Imaging and the American Society of Echocardiography. Eur Heart J Cardiovasc Imaging. 2013; 14(8): 721-740, indexed in Pubmed: 23847385.

18. Marlière $\mathrm{S}$, Vautrin $\mathrm{E}$, Saunier $\mathrm{C}$, et al. Radiation-related heart toxicity: Update in women. Ann Cardiol Angeiol (Paris). 2016; 65(6): 411-419, indexed in Pubmed: 27842711.

19. Carver JR, Shapiro CL, Ng A, et al. American Society of Clinical Oncology clinical evidence review on the ongoing care of adult cancer survivors: cardiac and pulmonary late effects. J Clin Oncol. 2007; 25(25): 3991-4008, doi: 10.1200/JCO.2007.10.9777, indexed in Pubmed: 17577017.

20. Kurowicki M, Zaucha R. Zastosowanie radioteraii w chłoniaku Hodgkina. Onkologia w praktyce klinicznej. 2014: 16-23.

21. McGale P, Darby SC, Hall P, et al. Incidence of heart disease in 35,000 women treated with radiotherapy for breast cancer in Denmark and Sweden. Radiother Oncol. 2011; 100(2): 167-175, doi: 10.1016/j.radonc.2011.06.016, indexed in Pubmed: 21752480.

22. Hull MC, Morris CG, Pepine CJ, et al. Valvular dysfunction and carotid, subclavian, and coronary artery disease in survivors of hodgkin lymphoma treated with radiation therapy. JAMA. 2003; 290(21): 2831-2837, doi: 10.1001/jama.290.21.2831, indexed in Pubmed: 14657067.

23. Annest LS, Anderson RP, Li W, et al. Coronary artery disease following mediastinal radiation therapy. J Thorac Cardiovasc Surg. 1983; 85(2): 257-263, indexed in Pubmed: 6823143.

24. Orzan F, Brusca A, Conte MR, et al. Severe coronary artery disease after radiation therapy of the chest and mediastinum: clinical presentation and treatment. Heart. 1993; 69(6): 496-500, doi: 10.1136/hrt.69.6.496.

25. Gyenes G, Fornander T, Carlens P, et al. Detection of radiationinduced myocardial damage by technetium-99m sestamibi scin- tigraphy. Eur J Nuclear Med. 1997; 24(3): 286-292, doi: 10.1007/ bf01728765.

26. Storey MR, Munden R, Strom EA, et al. Coronary artery dosimetry in intact left breast irradiation. Cancer J. 2001; 7(6): 492-497, indexed in Pubmed: 11769861.

27. Duane F, Aznar MC, Bartlett F, et al. A cardiac contouring atlas for radiotherapy. Radiother Oncol. 2017; 122(3): 416-422, doi:10.1016/j.radonc.2017.01.008, indexed in Pubmed: 28233564.

28. Heidenreich PA, Hancock SL, Lee BK, et al. Asymptomatic cardiac disease following mediastinal irradiation. J Am Coll Cardiol. 2003; 42(4): 743-749, indexed in Pubmed: 12932613.

29. Amromin GD, Gildenhorn HL, Solomon RD, et al. The synergism of $\mathrm{x}$-irradiation and cholesterol-fat feeding on the development of coronary artery lesions. J Atheroscl Res. 1964; 4(4): 325-334, doi: 10.1016/s0368-1319(64)80043-0.

30. Glanzmann C, Kaufmann P, Jenni R, et al. Cardiac risk after mediastinal irradiation for Hodgkin's disease. Radiotherapy Oncol. 1998; 46(1): 51-62, doi:10.1016/s0167-8140(97)00125-4.

31. Hahn E, Jiang H, Ng A, et al. Late cardiac toxicity after mediastinal radiation therapy for hodgkin lymphoma: contributions of coronary artery and whole heart dose-volume variables to risk prediction. Int J Radiat Oncol Biol Phys. 2017; 98(5): 1116-1123, doi: 10.1016/j.ijrobp.2017.03.026, indexed in Pubmed: 28721895.

32. Fleisher LA, Beckman JA, Brown KA, et al. ACC/AHA 2007 Guidelines on Perioperative Cardiovascular Evaluation and Care for Noncardiac Surgery: Executive Summary: A Report of the American College of Cardiology/American Heart Association Task Force on Practice Guidelines (Writing Committee to Revise the 2002 Guidelines on Perioperative Cardiovascular Evaluation for Noncardiac Surgery): Developed in Collaboration With the American Society of Echocardiography, American Society of Nuclear Cardiology, Heart Rhythm Society, Society of Cardiovascular Anesthesiologists, Society for Cardiovascular Angiography and Interventions, Society for Vascular Medicine and Biology, and Society for Vascular Surgery. Circulation. 2007; 116(17): 1971-1996, doi: 10.1161/CIRCULATIONAHA.107.185700, indexed in Pubmed: 17901356.

33. Iliescu C, Tsitlakidou D, Giza D, et al. Primary Percutaneous Coronary Interventions in Cancer Patients. Cancer Res Front. 2017; 3(1): 64-71, doi:10.17980/2017.64.

34. Heidenreich PA, Kapoor JR. Radiation induced heart disease: systemic disorders in heart disease. Heart. 2009; 95(3): 252-258, doi:10.1136/hrt.2008.149088, indexed in Pubmed: 19144884.

35. Herrmann J, Lerman A, Sandhu NP, et al. Evaluation and management of patients with heart disease and cancer: cardio-oncology. Mayo Clin Proc. 2014; 89(9): 1287-1306, doi: 10.1016/j. mayocp.2014.05.013, indexed in Pubmed: 25192616.

36. Saxena P, Joyce LD, Daly RC, et al. Cardiac transplantation for radiation-induced cardiomyopathy: the Mayo Clinic experience. Ann Thorac Surg. 2014; 98(6): 2115-2121, doi: 10.1016/j.athoracsur.2014.06.056, indexed in Pubmed: 25443015.

37. Donnellan E, Masri A, Johnston DR, et al. Long-term outcomes of patients with mediastinal radiation-associated severe aortic stenosis and subsequent surgical aortic valve replacement: a matched cohort study. J Am Heart Assoc. 2017; 6(5): e005396, doi: 10.1161/JAHA.116.005396, indexed in Pubmed: 28476874.

38. Fender EA, Chandrashekar P, Liang JJ, et al. Coronary artery bypass grafting in patients treated with thoracic radiation: a casecontrol study. Open Heart. 2018; 5(1): e000766, doi: 10.1136/ /openhrt-2017-000766, indexed in Pubmed: 29531769. 
39. Zhuang XF, Yang YM, Sun XL, et al. Late onset radiation-induced constrictive pericarditis and cardiomyopathy after radiotherapy: A case report. Medicine (Baltimore). 2017; 96(5): e5932, doi: 10.1097/ MD.0000000000005932, indexed in Pubmed: 28151876.

40. Fajardo LF, Stewart JR. Experimental radiation-induced heart disease. Light microscopic studies. Am J Pathol. 1970; 59(2): 299-316, indexed in Pubmed: 5443637.

41. Vaitkus P, Herrmann HC, LeWinter MM. Treatment of Malignant Pericardial Effusion. JAMA: J Am Med Assoc. 1994; 272(1): 59 , doi:10.1001/jama.1994.03520010071035.

42. El Haddad D, Iliescu C, Yusuf SW, et al. Outcomes of cancer patients undergoing percutaneous pericardiocentesis for pericardial effusion. J Am Coll Cardiol. 2015; 66(10): 1119-1128, doi: 10.1016/j.jacc.2015.06.1332, indexed in Pubmed: 26337990.

43. Nielsen KM, Offersen BV, Nielsen HM, et al. Short and long term radiation induced cardiovascular disease in patients with cancer. Clin Cardiol. 2017; 40(4): 255-261, doi: 10.1002/clc.22634, indexed in Pubmed: 28139844.

44. Wang K, Pearlstein KA, Patchett ND, et al. Heart dosimetric analysis of three types of cardiac toxicity in patients treated on dose-escalation trials for Stage III non-small-cell lung cancer. Radiother Oncol. 2017; 125(2): 293-300, doi: 10.1016/j.radonc.2017.10.001, indexed in Pubmed: 29050957.

45. Orzan F, Brusca A. Radiation-induced constrictive pericarditis. Associated cardiac lesions, therapy and follow-up. G Ital Cardiol. 1994; 24(7): 817-823, indexed in Pubmed: 7926379.

46. Syed FF, Schaff HV, Oh JK. Constrictive pericarditis: a curable diastolic heart failure. Nat Rev Cardiol. 2014; 11(9): 530-544, doi:10.1038/nrcardio.2014.100, indexed in Pubmed: 25072910.

47. Adler Y, Charron P, Imazio M, et al. 2015 ESC Guidelines for the diagnosis and management of pericardial diseases: The Task Force for the Diagnosis and Management of Pericardial Diseases of the European Society of Cardiology (ESC)Endorsed by: The European Association for Cardio-Thoracic Surgery (EACTS). Eur Heart J. 2015; 36(42): 2921-2964, doi: 10.1093/eurheartj/ /ehv318, indexed in Pubmed: 26320112.

48. Caforio ALP, Pankuweit S, Arbustini E, et al. Current state of knowledge on aetiology, diagnosis, management, and therapy of myocarditis: a position statement of the European Society of Cardiology Working Group on Myocardial and Pericardial Diseases. Eur Heart J. 2013; 34(33): 2636-2648, doi:10.1093/eurheartj/ /eht210, indexed in Pubmed: 23824828.

49. Hering D, Faber L, Horstkotte D. Echocardiographic features of Radiation-Associated valvular disease. Am J Cardiol. 2003; 92(2): 226-230, doi:10.1016/s0002-9149(03)00546-0.

50. Adams M, Hardenbergh P, Constine L, et al. Radiation-associated cardiovascular disease. Crit Rev Oncol Hematol. 2003; 45(1): 55-75, doi:10.1016/s1040-8428(01)00227-x.

51. Madan R, Benson R, Sharma DN, et al. Radiation induced heart disease: Pathogenesis, management and review literature. J Egypt Natl Canc Inst. 2015; 27(4): 187-193, doi: 10.1016/j. jnci.2015.07.005, indexed in Pubmed: 26296945.

52. Tuohinen SS, Skyttä T, Poutanen T, et al. Radiotherapy-induced global and regional differences in early-stage left-sided versus right-sided breast cancer patients: speckle tracking echocardiography study. Int J Cardiovasc Imaging. 2017; 33(4): 463-472, doi: 10.1007/s10554-016-1021-y, indexed in Pubmed: 27873127.

53. Kalam K, Otahal P, Marwick TH. Prognostic implications of global LV dysfunction: a systematic review and meta-analysis of global longitudinal strain and ejection fraction. Heart. 2014;
100(21): 1673-1680, doi: 10.1136/heartjnl-2014-305538, indexed in Pubmed: 24860005.

54. Umezawa $\mathrm{R}$, Ota $\mathrm{H}$, Takanami $\mathrm{K}$, et al. MRI findings of radiation-induced myocardial damage in patients with oesophageal cancer. Clin Radiol. 2014; 69(12): 1273-1279, doi: 10.1016/j. crad.2014.08.010, indexed in Pubmed: 25246336.

55. Skyttä T, Tuohinen S, Boman E, et al. Troponin T-release associates with cardiac radiation doses during adjuvant left-sided breast cancer radiotherapy. Radiat Oncol. 2015; 10: 141, doi: 10.1186/ /s13014-015-0436-2, indexed in Pubmed: 26159409.

56. Opolski G, Krzakowski M, Szmit S, et al. Recommendations of National Team of Cardiologic and Oncologic Supervision on cardiologic safety of patients with breast cancer. The prevention and treatment of cardiovascular complications in breast cancer. The Task Force of National Consultants in Cardiology. Kardiol Pol. 2011; 69(5): 520-530, indexed in Pubmed: 21594854.

57. Armenian SH, Lacchetti C, Barac A, et al. Prevention and Monitoring of Cardiac Dysfunction in Survivors of Adult Cancers: American Society of Clinical Oncology Clinical Practice Guideline. J Clin Oncol. 2017; 35(8): 893-911, doi: 10.1200/ JCO.2016.70.5400, indexed in Pubmed: 27918725.

58. Tadic M, Cuspidi C, Hering D, et al. Radiotherapy-induced right ventricular remodelling: the missing piece of the puzzle. Arch Cardiovasc Dis. 2017; 110(2): 116-123, doi: 10.1016/j. acvd.2016.10.003, indexed in Pubmed: 28117246.

59. Groarke JD, Nguyen PL, Nohria A, et al. Cardiovascular complications of radiation therapy for thoracic malignancies: the role for non-invasive imaging for detection of cardiovascular disease. Eur Heart J. 2014; 35(10): 612-623, doi: 10.1093/eurheartj/eht114, indexed in Pubmed: 23666251.

60. Grover S, Leong DP, Chakrabarty A, et al. Left and right ventricular effects of anthracycline and trastuzumab chemotherapy: a prospective study using novel cardiac imaging and biochemical markers. Int J Cardiol. 2013; 168(6): 5465-5467, doi: 10.1016/j. ijcard.2013.07.246, indexed in Pubmed: 24090744.

61. Tuohinen SS, Skyttä T, Virtanen V, et al. Detection of radiotherapy-induced myocardial changes by ultrasound tissue characterisation in patients with breast cancer. Int J Cardiovasc Imaging. 2016; 32(5): 767-776, doi: 10.1007/s10554-016-0837-9, indexed in Pubmed: 26757708.

62. Tadic M, Cuspidi C, Hering D, et al. The influence of chemotherapy on the right ventricle: did we forget something? Clin Cardiol. 2017; 40(7): 437-443, doi: 10.1002/clc.22672, indexed in Pubmed: 28191909.

63. Vedovati MC, Giustozzi M, Verdecchia P, et al. Patients with cancer and atrial fibrillation treated with doacs: A prospective cohort study. Int J Cardiol. 2018; 269: 152-157, doi: 10.1016/j. ijcard.2018.07.138, indexed in Pubmed: 30077526.

64. Laube ES, Yu A, Gupta D, et al. Rivaroxaban for stroke prevention in patients with nonvalvular atrial fibrillation and active cancer. Am J Cardiol. 2017; 120(2): 213-217, doi: 10.1016/j.amjcard.2017.04.009, indexed in Pubmed: 28549819.

65. Kalman PG, Lipton IH, Provan JL, et al. Radiation damage to large arteries. Can J Surg. 1983; 26(1): 88-91, indexed in Pubmed: 6821774.

66. Pherwani AD, Reid JA, Keane PF, et al. Synergism between radiotherapy and vascular risk factors in the accelerated development of atherosclerosis: a report of three cases. Ann Vasc Surg. 2002; 16(5): 671-675, doi: 10.1007/s10016-001-0117-5, indexed in Pubmed: 12183769. 
67. Bergqvist D, Jonsson K, Nilsson M, et al. Treatment of arterial lesions after radiation therapy. Surg Gynecol Obstet. 1987; 165(2): 116-1120, indexed in Pubmed: 3603340.

68. Fajardo LF. Berthrong M. Vascular lesions following radiation. Pathol Ann. 1988; 23(1): 297-330, indexed in Pubmed: 3387138.

69. Timmers HULM, Wieling W, Karemaker JM, et al. Baroreflex failure: a neglected type of secondary hypertension. Neth J Med. 2004; 62(5): 151-155, indexed in Pubmed: 15366697.

70. Farach A, Fernando R, Bhattacharjee M, et al. Baroreflex failure following radiotherapy for head and neck cancer: a case study. Pract Radiat Oncol. 2012; 2(3): 226-232, doi: 10.1016/j. prro.2011.06.006, indexed in Pubmed: 24674125.

71. Goldstein DS, Cheshire WP. Beat-to-beat blood pressure and heart rate responses to the Valsalva maneuver. Clin Auton Res. 2017; 27(6): 361-367, doi: 10.1007/s10286-017-0474-y, indexed in Pubmed: 29052077.

72. Goyal M, Shukla P, Gupta D, et al. Cardiovascular sequel of neck irradiation in head and neck cancer patients. Int J Radiat Biol. 2017; 93(7): 711-716, doi: 10.1080/09553002.2017.1303217, indexed in Pubmed: 28376642.

73. Huang CC, Huang TL, Hsu HC, et al. Long-term effects of neck irradiation on cardiovascular autonomic function: a study in nasopharyngeal carcinoma patients after radiotherapy. Muscle Nerve. 2013; 47(3): 344-350, doi: 10.1002/mus.23530, indexed in Pubmed: 23386577.
74. Timmers HJ, Karemaker JM, Lenders JW, et al. Baroreflex failure following radiation therapy for nasopharyngeal carcinoma. Clin Auton Res. 1999; 9(6): 317-324, doi: 10.1007/BF02318378, indexed in Pubmed: 10638805.

75. Goldstein DS. Neurocardiology: therapeutic implications for cardiovascular disease. Cardiovasc Ther. 2012; 30(2): e89-106, doi: 10.1111/j.1755-5922.2010.00244.x, indexed in Pubmed: 21108771.

76. Krupicka J, Marková J, Pohlreich D, et al. Echocardiographic evaluation of acute cardiotoxicity in the treatment of Hodgkin disease according to the German Hodgkin's Lymphoma Study Group. Leuk Lymphoma. 2002; 43(12): 2325-2329, indexed in Pubmed: 12613519.

77. Andratschke N, Maurer J, Molls M, et al. Late radiation-induced heart disease after radiotherapy. Clinical importance, radiobiological mechanisms and strategies of prevention. Radiother Oncol. 2011; 100(2): 160-166, doi: 10.1016/j.radonc.2010.08.010, indexed in Pubmed: 20826032.

78. Senkus-Konefka E, Jassem J. Cardiovascular effects of breast cancer radiotherapy. Cancer Treat Rev. 2007; 33(6): 578-593, doi: 10.1016/j.ctrv.2007.07.011, indexed in Pubmed: 17764850.

79. Guddati AK, Joy PS, Kumar G. Analysis of outcomes of percutaneous coronary intervention in metastatic cancer patients with acute coronary syndrome over a 10 -year period. J Cancer Res Clin Oncol. 2016; 142(2): 471-479, doi: 10.1007/s00432-0152056-5, indexed in Pubmed: 26498773. 